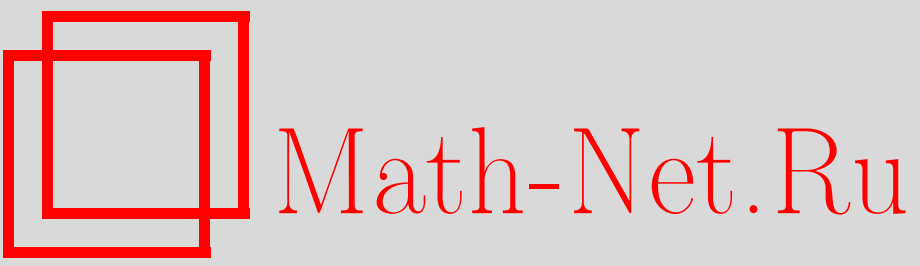

О. И. Завьялов, О механизме появления нелинейных представлений группы Лоренца в квантовой теории поля, ТМФ, 2001, том 128, номер 3, 403-408

DOI: https://doi.org/10.4213/tmf504

Использование Общероссийского математического портала Math-Net.Ru подразумевает, что вы прочитали и согласны с пользовательским соглашением

http://www . mathnet.ru/rus/agreement

Параметры загрузки:

IP : 54.224 .60 .19

26 апреля 2023 г., 15:14:25 
ТЕОРЕТИЧЕСКАЯ

И МАТЕМАТИЧЕСКАЯ

ФИЗИКА

Том 128, № 3

сентябрь, 2001

(C) 2001 г.

О.И. Завьялов*

\section{О МЕХАНИЗМЕ ПОЯВЛЕНИЯ НЕЛИНЕЙНЫХ ПРЕДСТАВЛЕНИЙ ГРУППЫ ЛОРЕНЦА В КВАНТОВОЙ ТЕОРИИ ПОЛЯ}

Выявлен "механизм", в силу которого релятивистская функция Вигнера имеет "правильный" закон преобразования по отношению к группе Лоренца, только если соответствующие релятивистские волновые функции обладают специальными трансформационными свойствами.

Эту статью я посвящаю Владимиру Яковлевичу Файнбергу, блестящие лекции которого по классической электродинамике я имел удовольствие слушать еще в свои студенческие годы и который уже в те (увы, давние) времена олицетворял для меня идеальный образ настоящего мужчины (физика-теоретика).

\section{1. ВВЕДЕНИЕ}

Данная работа является прямым продолжением статей [1] и [2]. В них приведены соображения, указывающие на то, что преобразования (по отношению к группе Лоренца) релятивистских волновых функций свободных частиц (если квантовая механика таких частиц является самосогласованной) должны быть нелинейными. Ниже показано, как может возникнуть эта нелинейность. В [1] было установлено, что при изменении лоренцевой системы отсчета функция Вигнера свободных частиц изменяется только за счет импульсной переменной $\gamma$, причем соответствующему преобразования сдвига $(\gamma \rightarrow \gamma+\nu)$ подвергается лишь $\gamma$, которая не входит в “координатный” множитель $\exp (i q \operatorname{sh} \gamma)$ при волновой функции $\psi(\gamma)$. Именно,

$$
W(\gamma, q ; 0) \rightarrow W_{q}(\gamma+\nu, q ; 0)
$$

где $\nu$ - относительная скорость систем отсчета, а индекс $q$ означает здесь, что переменная $\gamma$, фигурируюшая помимо переменной $q$ в указанном "координатном" множителе, остается постоянной и не подвергается сдвигу в (1) (в статье [1] это была формула (49)).

\footnotetext{
* Математический институт им. В. А. Стеклова РАН, Москва, Россия
} 
В статье [1] мы привели косвенные аргументы в пользу того, что нелинейность преобразований волновых функций имеет место и при ненулевых $q$. В данной статье мы превратим эти полуэвристические аргументы в технически последовательное доказательство.

Далее мы будем использовать методы, практически совпадающие с методами работ [1], [2]. Для того чтобы ввести адекватные этим методам термины в новой ситуации с ненулевыми $q$, мы все же явно выпишем здесь важную исходную формулу работы [1], выражающую вигнеровское распределение через волновую функцию $\psi_{q}(\gamma)=$ $\psi(\gamma) \exp (i q \operatorname{sh} \gamma)$, где импульс $p$, первоначально входящий в волновую функцию $\psi$ свободной частицы, есть $p=\operatorname{sh} \gamma$. Именно,

$$
W(\gamma, q ; 0)=\int d \gamma^{\prime} d \gamma^{\prime \prime} \frac{\operatorname{ch}\left(\gamma^{\prime}\right) \operatorname{ch}\left(\gamma^{\prime \prime}\right)}{2 \pi \operatorname{ch} \gamma} \psi_{q}^{*}\left(\gamma^{\prime}\right) \psi_{q}\left(\gamma^{\prime \prime}\right) \delta\left(\gamma-\frac{\gamma^{\prime}+\gamma^{\prime \prime}}{2}\right)
$$

В работе [1] эта формула имела номер (40).

Фурье-преобразование $\widetilde{W}_{q}(\xi)$ функции $W(\gamma, q ; 0)$ определено соотношением

$$
\widetilde{W}_{q}(\xi)=\int d \gamma \exp (i \xi \gamma) \operatorname{ch} \gamma W(\gamma, q ; 0)
$$

Обратное к (2) преобразование имеет вид

$$
W(\gamma, q ; 0)=\frac{1}{2 \pi \operatorname{ch} \gamma} \int d \xi \exp (-i \xi \gamma) \widetilde{W}_{q}(\xi)
$$

Как и ранее, это соотношение означает, что

$$
\widetilde{W}_{q}(\xi)=\frac{1}{2 \pi} \widetilde{\psi}_{q}^{*}\left(-\frac{\xi}{2}\right) \widetilde{\psi}_{q}\left(\frac{\xi}{2}\right)
$$

причем

$$
\widetilde{\psi}_{q}(\xi)=\int d \gamma \exp (i \gamma \xi) \psi_{q}(\gamma) \operatorname{ch} \gamma
$$

\section{2. ПРЕОБРАЗОВАНИЯ ВОЛНОВЫХ ФУНКЦИЙ}

Согласно соотношениям (1) и (2) условие лоренц-инвариантности функции Вигнера при произвольных $q$ имеет во введенных обозначениях следующий стандартньй для наших рассмотрений вид:

$$
\widetilde{W}(\xi) \rightarrow \widetilde{W}_{q}^{\nu}(\xi)=\widetilde{W}_{q}+\delta \widetilde{W}_{q}=\widetilde{W}_{q}(\xi)(1-i \xi \nu)-\nu \int d \eta \Delta(\xi-\eta) \widetilde{W}_{q}(\eta)
$$

(последнее равенство, разумеется, справедливо только инфинитезимально). Напомним также, что

$$
\Delta(\xi)=\int \frac{d \gamma}{2 \pi} \operatorname{th} \gamma \exp (i \xi \gamma)
$$


(это формула (30) работы [2]). Теперь можно, пользуясь условием (4) и фактически повторяя рассуждения статей [1], [2], получить формулы, задаюшие (инфинитезимальные) преобразования (по отношению к лоренцевым бустам $\nu$ ) для волновых функций $\widetilde{\psi}_{q}(\xi)$. Именно, мы утверждаем, что условие лоренц-инвариантности функции Вигнера будет выполнено, если при малом лоренцевом повороте с относительной скоростью $\nu$ волновая функция будет изменяться по правилу

$$
\widetilde{\psi}_{q}(\xi) \rightarrow \widetilde{\psi}_{q}^{\nu}(\xi)=\widetilde{\psi}_{q}(\xi)+\delta \widetilde{\psi}_{q}(\xi)=\widetilde{\psi}_{q}(\xi)-\nu \widetilde{\Phi}_{q}(\xi) \widetilde{\psi}_{q}(\xi)
$$

При этом

$$
\widetilde{\Phi}_{q}(\xi)=\widetilde{S}_{q}(\xi)+\widetilde{A}_{q}(\xi)=i \xi+\int d \eta \frac{1}{\widetilde{W}_{q}(2 \xi)} \Delta(2 \xi-2 \eta) \widetilde{W}_{q}(2 \eta)+\widetilde{A}_{q}(\xi)
$$

так что $\widetilde{S}_{q}(\xi)$ - квазивешественная часть функции $\widetilde{\Phi}_{q}(\xi)$, а квазимнимая часть $\widetilde{A}_{q}(\xi)$ этой функции произвольна. Обозначения $\widetilde{S}$ и $\widetilde{A}$ связаны с терминами “симметричная" и “антисимметрическая" части функции $\widetilde{\Phi}$ по отношению к введенной в [2] квазиинволюции (которая, напомним, соответствует обычному комплексному сопряжению для фурье-образов этих функций). Разумеется, фигурирующая в $(8)$ квазимнимая часть $\widetilde{A}_{q}(\xi)$ может, вообше говоря, зависеть как от $q$, так и от самой исходной волновой функции $\psi(\gamma)$

Приведем еще и в этой статье сокрашенное доказельство сформулированного только что важного утверждения о лоренц-инвариантности величины $W(\gamma, q ; 0)$. Прежде всего снова упомянем о том, что вклад от квазимнимой функции $\widetilde{A}_{q}(\xi)$, входяшей (через $\widetilde{\Phi}_{q}(\xi)$ ) из $(7)$ в вариацию $\delta \widetilde{\psi}_{q}(\xi)$, выпадает (в силу соотношения $\left.\widetilde{A}_{q}^{*}(-\xi)=-\widetilde{A}_{q}(\xi)\right)$ из вариации $\delta W(\gamma, q ; 0)$. Таким образом, в $\widetilde{\Phi}(\xi)$ можно сразу положить $\widetilde{A}_{q}(\xi)=0$. В этом случае будет справедливо равенство

$$
\widetilde{\Phi}_{q}(\xi)=\widetilde{\Phi}_{1}(\xi)+\widetilde{\Phi}_{2}(\xi)
$$

где

$$
\widetilde{\Phi}_{1}(\xi)=i \xi, \quad \widetilde{\Phi}_{2}(\xi)=\int d \eta \frac{1}{\widetilde{W}_{q}(2 \xi)} \Delta(2 \xi-2 \eta) \widetilde{W}_{q}(2 \eta)
$$

Каждое из этих двух квазивешественных слагаемых порождает свой вклад $\delta_{1} \widetilde{\psi}_{q}(\xi)$ или $\delta_{2} \widetilde{\psi}_{q}(\xi)$ в вариацию $\delta \widetilde{\psi}_{q}(\xi)$ и соответственно свой вклад $\delta_{1} \widetilde{W}_{q}(\xi)$ или $\delta_{2} \widetilde{W}_{q}(\xi)$ в вариацию $\delta \widetilde{W}_{q}(\xi)$. При этом в первом порядке по $\nu$ вклад $\delta_{2} \widetilde{W}_{q}(2 \xi)$ будет содержать член $\widetilde{\Phi}(2 \xi) \widetilde{\psi}_{q}^{*}(-\xi) \widetilde{\psi}_{q}(\xi)$, так что благодаря соотношению (4) фактор $\widetilde{W}_{q}(2 \xi)$, стояший в знаменателе формулы (10), сократится. Таким образом,

$$
\delta_{1} \widetilde{W}_{q}(2 \xi)=-2 \nu \xi \widetilde{W}_{q}(2 \xi)
$$

и

$$
\delta_{2} \widetilde{W}_{q}(2 \xi)=-\nu \int d \eta \Delta(2 \xi-\eta) \widetilde{W}_{q}(\eta)
$$


Окончательно будем иметь

$$
\delta \widetilde{W}_{q}(2 \xi)=-2 i \nu \xi \widetilde{W}_{q}(2 \xi)-\nu \int d \eta \Delta(2 \xi-\eta) \widetilde{W}_{q}(\eta)
$$

что и является условием лоренц-инвариантности для функции $\widetilde{W}_{q}(2 \xi)$.

Свяжем теперь допустимую вариацию координатной волновой функции $\delta \widetilde{\psi}_{q}(\xi)$, фигурируюшую в (7), с вариацией собственно волновой функции

$$
\delta \widetilde{\psi}(\xi)=\delta \int d \gamma \exp (i \gamma \xi) \operatorname{ch} \gamma \psi(\gamma)
$$

Поскольку по нашему предположению переменные, входящие в "координатный" множитель, никак не преобразуются при лоренцевых поворотах, то именно эта последняя величина и является независимой, целиком определяя все другие вариации. В частности,

$$
\delta \widetilde{\psi}_{q}(\xi)=\int d \eta \widetilde{D}_{q}(\xi-\eta) \delta \widetilde{\psi}(\eta)
$$

где

$$
\widetilde{D}_{q}(\xi)=\int \frac{d \gamma}{2 \pi} \exp (i \gamma \xi) \exp (i q \operatorname{sh} \gamma)
$$

(последнее равенство, конечно, нужно понимать в смысле обобщенных функций). Имеем

$$
\lim _{q \rightarrow 0} \widetilde{D}_{q}(\xi)=\delta(\xi)
$$

Соотношение (14), по сушеству, использует тот факт, что величина $\psi_{q}(\gamma)$ представляет собой сдвиг функции $\psi(\gamma)$ на вектор $q$, т.е.

$$
\widetilde{\psi}_{q}(\xi)=\int d \eta \widetilde{D}_{q}(\xi-\eta) \widetilde{\psi}(\eta)
$$

Отметим также соотношение

$$
\int d \eta \widetilde{D}_{-q}(\xi-\eta) \widetilde{D}_{q}\left(\eta-\xi^{\prime}\right)=\delta\left(\xi-\xi^{\prime}\right)
$$

так что равенства, обратные к (14) и (16), имеют вид

$$
\delta \widetilde{\psi}(\xi)=\int d \eta \widetilde{D}_{-q}(\xi-\eta) \delta \widetilde{\psi}_{q}(\eta)
$$

и

$$
\widetilde{\psi}(\xi)=\int d \eta \widetilde{D}_{-q}(\xi-\eta) \widetilde{\psi}_{q}(\eta)
$$


Таким образом, с учетом закона трансформации (см. формулу (7)) в терминах нашего основного объекта $\delta \widetilde{\psi}(\xi)$ получаем правило

$$
\begin{aligned}
\delta \widetilde{\psi}(\xi) & =-\nu \int d \eta_{1} \widetilde{D}_{-q}\left(\xi-\eta_{1}\right) \widetilde{\Phi}_{q}\left(\eta_{1}\right) \widetilde{\psi}_{q}\left(\eta_{1}\right)= \\
& =-\nu \int d \eta_{1} d \eta_{2} \widetilde{D}_{-q}\left(\xi-\eta_{1}\right) \widetilde{\Phi}_{q}\left(\eta_{1}\right) \widetilde{D}_{q}\left(\eta_{1}-\eta_{2}\right) \widetilde{\psi}\left(\eta_{2}\right) .
\end{aligned}
$$

Теперь нам осталось доказать, что вся совокупность преобразований (20) (при различных $q$ ) сводится фактически к единому преобразованию в том смысле, что все они согласованы друг с другом и, в частности, со стандартным исходным преобразованием при $q=0$, которое впервые было получено в работе [2]. Иными словами, нам предстоит убедиться, что существует способ выбрать квазимнимые функции $\widetilde{A}_{q}(\xi)$ такие, что для всех функций $\psi(\gamma)$ и всех $q$ будет выполнено равенство

$$
\int d \eta_{1} d \eta_{2} \widetilde{D}_{-q}\left(\xi-\eta_{1}\right) \widetilde{\Phi}_{q}\left(\eta_{1}\right) \widetilde{D}_{q}\left(\eta_{1}-\eta_{2}\right) \widetilde{\psi}\left(\eta_{2}\right)=\widetilde{\Phi}_{0}(\xi) \widetilde{\psi}(\xi)
$$

Это ключевое равенство, разумеется, будет выполнено, если удастся удовлетворить уравнению

$$
\int d \lambda \widetilde{D}_{-q}(\xi-\lambda) \widetilde{\Phi}_{q}(\lambda) \widetilde{D}_{q}(\lambda-\eta)=\widetilde{\Phi}_{0}(\xi) \delta(\xi-\eta) .
$$

Решение этого уравнения имеет вид

$$
\widetilde{\Phi}_{q}(\lambda) \delta(\lambda-\eta)=\int d \beta \widetilde{D}_{q}(\lambda-\beta) \widetilde{\Phi}_{0}(\beta) \widetilde{D}_{-q}(\beta-\eta) .
$$

С учетом базовых свойств функции Вигнера [3], доказанных в [4], можно утверждать, что интеграл в правой части соотношения (23) удовлетворяет своеобразной “теореме о среднем". Точнее говоря, сушествует такая функция $\beta_{q, A_{0}}^{*}(\lambda, \eta)$, что величину $\widetilde{\Phi}_{0}$, взятую в фиксированной точке $\beta=\beta^{*}$, можно вынести за знак этого интеграла. Возьмем теперь произвольную основную функцию $\varphi(\eta)$, которая, однако, нигде на вешественной оси не обрашается в нуль, и проинтегрируем с этим весом равенство (23). Находим

$$
\widetilde{\Phi}_{q}(\lambda)=\int d \eta d \beta \widetilde{D}_{q}(\lambda-\beta) \widetilde{\Phi}_{0}(\beta) \widetilde{D}_{-q}(\beta-\eta) \varphi(\eta)[\varphi(\lambda)]^{-1} .
$$

Очевидно, что правая часть (24) в рассматриваемой ситуации не зависит ни от $\lambda$, ни от самой $\varphi$ и что на самом деле равенство (24) сводится к равенству

$$
\widetilde{\Phi}_{q}(\lambda)=\widetilde{\Phi}_{0}\left(\beta_{q, A_{0}}^{*}(\lambda, \lambda)\right)
$$

В найденном нами решении есть произвол, связанный с неоднозначностью выбора квазимнимой функции $\widetilde{A}_{0}(\xi)$, содержашейся в $\widetilde{\Phi}_{0}(\xi)$ (в обозначениях работы [2] - это та самая квазимнимая функция $A(\xi)$, которую еше нужно было найти после рассмотрения 
случая $q=0$, исследованного в этой работе). Указанным произволом нужно распорядиться таким образом, чтобы квазивешественная часть $\widetilde{S}_{q}(\xi)$ функции $\widetilde{\Phi}_{q}(\xi)$ имела надлежаший вид (8). Дело здесь в том, что наличие в $\widetilde{\Phi}_{0}(\xi)$ квазимнимой компоненты в сочетаниях с ненулевыми функциями $\widetilde{D}_{q}$ и $\widetilde{D}_{-q}$ сказывается не только на квазимнимой составляющей величины $\widetilde{\Phi}_{q}(\xi)$, но и на ее квазивешественной составляюшей. Определяя величину $\widetilde{A}_{0}(\xi)$ по $(23)$ из условия совпадения квазивешественной части функции $\widetilde{\Phi}_{q}(\xi)$ с правой частью соотношения (9) (см. также (10)), мы найдем тем самым и нужную нам функцию $\widetilde{A}_{q}(\xi)$ как квазимнимую часть величины $\widetilde{\Phi}_{q}(\xi)$ при этой избранной функции $\widetilde{A}_{0}(\xi)$. К сожалению, мы должны ограничиться сейчас этими общими соображениями типа “теоремы существования", так как нам не удалось написать сколько-нибудь компактные явные формулы, решаюшие эту сложную задачу.

Благодарности. Работа была поддержана Российским фондом фундаментальных исследований (грант № 01-01-01043).

\section{Список литературы}

[1] О.И. Завьялов. ТМФ. 2001. Т. 127. № 1. С. 75 .

[2] О. И. Заввялов. Труды МИАН им. В. А. Стеклова. 2000. Т. 228. С. 136.

[3] E. Wigner. Phys. Rev. 1932. V. 40. P. 749.

[4] О.И. Завьялов, А. М. Малокостов. ТМФ. 1999. Т. 119. С. 67.

Поступила в редакцию 20.IV.2001 г. 